\title{
Robust Consensus for Nonlinear Multiagent Systems with Uncertainty and Disturbance
}

\author{
Zhiqiang Li, ${ }^{1}$ Chengjie Xu $\left(D,{ }^{2,3}\right.$ Chen Liu, ${ }^{2}$ and Haichuan $X u^{2}$ \\ ${ }^{1}$ School of Computer Science and Engineering, Yulin Normal University, Yulin 537000, China \\ ${ }^{2}$ School of Science, Hunan University of Technology, Zhuzhou 412007, China \\ ${ }^{3}$ School of Automation, Huazhong University of Science and Technology, Wuhan 430074, China \\ Correspondence should be addressed to Chengjie Xu; xu-chengjie@163.com
}

Received 5 March 2018; Revised 25 May 2018; Accepted 19 June 2018; Published 17 July 2018

Academic Editor: Luis Gracia

Copyright (C) 2018 Zhiqiang Li et al. This is an open access article distributed under the Creative Commons Attribution License, which permits unrestricted use, distribution, and reproduction in any medium, provided the original work is properly cited.

\begin{abstract}
This paper investigates robust consensus for nonlinear multiagent systems with uncertainty and disturbance. The consensus evolution behavior is studied under general consensus protocol when each node is disturbed by the relative states between the node and its neighbors. At first, the robust consensus condition is obtained and the convergency analysis is given by using Lyapunov stability theory and matrix theory. Then, the practical consensus is investigated and the bound of the error states is presented. Finally, two numerical simulation examples are given to illustrate the proposed theoretical results.
\end{abstract}

\section{Introduction}

In the past few years, increasing attention has been devoted to the coordination of multiagent systems due to its wide applications for sensor network and multirobot systems [1]. As one of the most important problems in the issue of distributed coordination, consensus has even attracted much more interest from researchers. And many profound results are reported [2-6].

Consensus means that the states of the agents reach an agreement on a common physical quantity by implementing an appropriate consensus protocol based on the information from local neighbors [7], which is mainly influenced by the topology of the network and the dynamics of each node. Thereby, on one side, many works about consensus of multiagent systems under switching topology are reported [8-10]. On the other side, consensus of multiagent systems with nonlinear dynamics or disturbances is intensively investigated [11-15]. External disturbance widely exists in real processes and is a main source of instability and poor performance. When any of the nodes is disturbed, coordinated behavior will be destroyed. Thereby, it is of great significance to investigate distributed coordination for nonlinear multiagent systems with bounded disturbances or stochastic disturbances. Mean square average consensus is investigated for multiagent systems with noisy measurement under directed topology. Necessary and sufficient consensus conditions are established [16]. Mean square leader-following consensus for multiagent systems with noisy channels under directed switching topology is investigated [17]. Necessary and sufficient average consensus conditions are established for multiple doubleintegrator systems with noisy measurement. It is proven that mean square average consensus can be realized if and only if the topology is balanced and strongly connected [18]. Using disturbance observer method, robust leader-following consensus was investigated for nonlinear coupled multiagent systems with external disturbance [19]. Containment control problem is studied for general linear multiagent systems with exogenous disturbances. Both the state feedback and output feedback containment protocol are proposed by using the disturbance observer approach [20]. Using sliding-mode control method, finite consensus and containment were investigated for second-order nonlinear multiagent systems with disturbances under directed topology [21]. Bounded consensus tracking is investigated for linear multiagent systems with actuator saturation and input additive uncertainties and disturbances based on low-and-high gain feedback approach [22].

However, few works investigate the consensus behavior when the relative information of the subsystem is disturbed, 
which widely exists in the real world. Therefore, in this paper, the consensus evolution behavior is investigated for nonlinear multiagent systems with uncertainty and disturbance. The main contribution in this paper is that the disturbance considered is dependent on the addition of the relative states between each node and their neighbors, and the disturbance satisfies a very mild condition, which is more relaxed than the condition in $[21,22]$. The uncertainty and disturbance function is described as a piecewise continuous function $g(x, t)$, satisfying $\|g(x, t)\|_{2} \leq g_{0}\left(\|x\|_{2}\right)+D_{0}$. Both robust consensus and practical consensus condition are obtained for $D_{0}=0$ and $D_{0} \neq 0$, respectively. This leads to the fact that the problems investigated in this paper are theoretically challenging and practically important.

The rest of the paper is organized as follows. Section 2 states the model considered in the paper and gives some basic definitions, lemmas, and assumptions. In Section 3, complete consensus protocol is proposed and the convergency analysis is given and practical consensus protocol is obtained in Section 4. In Section 5, two numerical simulation examples are given for illustrating the theoretical results. Finally, Section 6 concludes the paper.

\section{Preliminaries and Model Description}

A networked multiagent system under undirected topology consists of many agents and their communication, which can be described as an undirected graph $\mathscr{G}=(\mathscr{V}, \mathscr{E}, \mathscr{A})$. The set of nodes $\mathscr{V}=\left(v_{1}, v_{2}, \ldots, v_{N}\right)$ denotes the agents. The set of edges $\mathscr{E} \subseteq \mathscr{V} \times \mathscr{V}$ denotes communication relation of the agents. $\mathscr{A}=\left[a_{i j}\right]$ is the adjacent matrix of the nodes, which is defined as follows: $a_{i j}=1 \Longleftrightarrow\left(v_{j}, v_{i}\right) \in \mathscr{E}$; otherwise $a_{i j}=0$. A path is a sequence of edges in a directed graph of the form $\left(\vartheta_{1}, \vartheta_{2}\right),\left(\vartheta_{2}, \vartheta_{3}\right), \ldots$, where $\vartheta_{j} \in \mathscr{V}$. An undirected graph is connected if there is a path between any two nodes. The Laplacian matrix $\mathscr{L}=\left[l_{i j}\right] \in R^{N \times N}$ is defined as $l_{i i}=$ $\sum_{j=1, j \neq i}^{N} a_{i j}$ and $l_{i j}=-a_{i j}, i \neq j$.

Assume that there are $N$ follower agents, labeled as $1,2, \ldots, N$. The dynamics of the $i$ th agent are described as

$$
\dot{x}_{i}(t)=f\left(t, x_{i}\right)+u_{i}(t), \quad i=1,2, \ldots, N
$$

where $x_{i}, u_{i} \in R^{n}$ are the position and the input vector of the ith follower, respectively. $f: R \times R^{n} \longrightarrow R^{n}, i=1,2, \ldots, k$, is a continuous vector-value function, which describes the intrinsic local dynamics of the ith node.

The following assumption is necessary for our main results.

Assumption 1. Assume that $G$ is connected.

Assumption 2. Suppose that $f(t, x)$ satisfies the Lipschitz condition; that is, for arbitrary $x_{1}, x_{2}$, there exists a constant $\eta$ such that

$$
\begin{aligned}
\left\|f\left(t, x_{1}\right)-f\left(t, x_{2}\right)\right\|_{2} \leq \eta\left\|x_{1}-x_{2}\right\|_{2}, & \\
& \forall x_{1}, x_{2} \in R^{N} .
\end{aligned}
$$

Lemma 3 (see [2]). If $L=\left(l_{i j}\right) \in R^{N \times N}$ is a symmetric irreducible matrix with $l_{i i}=-\sum_{j=1, j \neq i}^{N} l_{i j}, l_{i j}=l_{j i} \leq 0(i \neq$ $j)$, then $L$ is semipositive definite, and for any matrix $H=$ $\operatorname{diag}(h, 0, \cdots, 0)$ with $h>0$, all eigenvalues of the matrix $(L+H)$ are positive.

Lemma 4 (see [23]). For an undirected connected graph $G$ with the Laplacian matrix $L$ and the vector $x$ satisfying $\mathbf{1}^{T} x=$ 0 ,

$$
\min _{x \neq 0}\left\{\frac{x^{T} L x}{x^{T} x}\right\}=\lambda_{2}(L) .
$$

\section{Robust Leader-Following Consensus for Nonlinear Multiagent Systems with Uncertainty and Disturbance}

In this section, the robust leader-following consensus of nonlinear multiagent systems with bounded channel disturbance is investigated. Suppose that there is a virtual leader in the network, whose dynamics are described as

$$
\dot{x}_{0}(t)=f\left(t, x_{0}\right) \text {. }
$$

Consider the following consensus protocol with the channel disturbance:

$$
\begin{gathered}
u_{i}=\sum_{j \in \mathcal{N}_{i}} a_{i j}\left[\alpha\left(x_{j}-x_{i}\right)+g\left(x_{j}-x_{i}, t\right)\right] \\
+\alpha h_{i}\left(x_{0}-x_{i}\right), \quad i=1,2, \ldots, N
\end{gathered}
$$

where $\alpha>0$ denotes the control gain to be determined; the nonlinear function $g: R^{n} \times R \longrightarrow R^{n}$ denotes the actuator input additive uncertainty and disturbance, satisfying $g\left(x_{j}-\right.$ $\left.x_{i}, t\right)=-g\left(x_{i}-x_{j}, t\right)$.

Assumption 5. The channel disturbance function $g(x, t)$ is piecewise continuous in $t$ and locally Lipschitz in $x$ and its norm is bounded by a known function.

$$
\|g(x, t)\|_{2} \leq g_{0}\left(\|x\|_{2}\right)+D_{0}, \quad \forall(x, t) \in R^{n} \times R_{+},
$$

where $g_{0}: R_{+} \longrightarrow R_{+}$is locally Lipschitz and satisfies $g_{0}(0)=$ 0 and $D_{0}$ is a positive constant.

Remark 6. For a given positive constant $\delta$, define

$$
M_{\delta}=\sup \left\{\frac{g_{0}(s)}{s} \mid s \in(0, \delta)\right\} \text {. }
$$

Under protocol (5), (1) can be rewritten as

$$
\begin{aligned}
\dot{x}_{i}(t)= & f\left(t, x_{i}\right) \\
& +\sum_{j \in \mathcal{N}_{i}} a_{i j}\left[\alpha\left(x_{j}-x_{i}\right)+g\left(x_{j}-x_{i}, t\right)\right] \\
& +\alpha h_{i}\left(x_{0}-x_{i}\right), \quad i=1,2, \ldots, N .
\end{aligned}
$$


Let $e_{i}=x_{i}-x_{0}$; then

$$
\begin{aligned}
\dot{e}_{i}(t)= & f\left(t, x_{i}\right)-f\left(t, x_{0}\right)+u_{i} \\
= & f\left(t, x_{i}\right)-f\left(t, x_{0}\right)+\alpha h_{i}\left(x_{0}-x_{i}\right) \\
& +\sum_{j \in \mathcal{N}_{i}} a_{i j}\left[\alpha\left(x_{j}-x_{i}\right)+g\left(x_{j}-x_{i}, t\right)\right] \\
= & f\left(t, x_{i}\right)-f\left(t, x_{0}\right)-\sum_{j \in \mathcal{N}_{i}} \alpha l_{i j} e_{j}-\alpha h_{i} e_{i} \\
& +\sum_{j \in \mathcal{N}_{i}} a_{i j} g\left(x_{j}-x_{i}, t\right), \quad i=1,2, \ldots, N .
\end{aligned}
$$

Lemma 7 (see [22]). For any $e_{i}, e_{j} \in R^{n}, i, j=1,2, \ldots, N$,

$$
\begin{aligned}
\frac{1}{2} \sum_{i=1}^{N} \sum_{j=1}^{N} a_{i j} e_{i}^{T} g\left(x_{j}-x_{i}, t\right) \\
\quad=-\sum_{i=1}^{N} \sum_{j=1}^{N} a_{i j}\left(e_{j}-e_{i}\right)^{T} g\left(x_{j}-x_{i}, t\right) .
\end{aligned}
$$

Lemma 8 (see [24]). For any $e_{i}, e_{j} \in R^{n}, i, j=1,2, \ldots, N$,

$$
\frac{1}{2} \sum_{i=1}^{N} \sum_{j=1}^{N} a_{i j}\left(e_{j}-e_{i}\right)^{T}\left(e_{j}-e_{i}\right)=\sum_{i=1}^{N} \sum_{j=1}^{N} l_{i j} e_{i}^{T} e_{j}
$$

3.1. Robust Leader-Following Consensus. In this subsection, robust consensus for nonlinear multiagent systems is investigated in the case where $D_{0}=0$ in Assumption 5 .

Theorem 9. Consider a networked multiagent system with $N$ followers and a virtual leader, in which the dynamics are described as (1)-(4). Suppose that Assumptions 1-5 hold and the constant $D_{0}=0$ in Assumption 5. Under the consensus protocol (5), robust consensus of system (1) can be achieved if the following two conditions hold:

(i) There exists at least one pinned node.

(ii) For a given positive constant $\delta$,

$$
\left(\alpha-4 M_{\delta}\right) \lambda_{1}>\eta
$$

where $\lambda_{1}$ is the minimal eigenvalue of $(L+H)$.

Furthermore, all the following nodes will track the virtual leader asymptotically.

Proof. Choose the Lyapunov candidate function as

$$
V=\frac{1}{2} \sum_{i=1}^{N} e_{i}^{T} e_{i}
$$

Differentiating $V$ with respect to $t$ along (9), one can obtain

$$
\begin{aligned}
\dot{V}= & \sum_{i=1}^{N} e_{i}^{T} \dot{e}_{i} \\
= & \sum_{i=1}^{N} e_{i}^{T}\left(f\left(t, x_{i}\right)-f\left(t, x_{0}\right)\right) \\
& -\sum_{i=1}^{N} e_{i}^{T}\left(\sum_{j=1}^{N} \alpha l_{i j} e_{j}+\alpha h_{i} e_{i}-\sum_{j=1}^{N} a_{i j} g\left(x_{j}-x_{i}, t\right)\right) \\
\leq & \eta \sum_{i=1}^{N} e_{i}^{T} e_{i}-\alpha \sum_{i=1}^{N} \sum_{j=1}^{N} e_{i}^{T} l_{i j} e_{j}-\alpha h_{i} \sum_{i=1}^{N} e_{i}^{T} e_{i} \\
& +\sum_{i=1}^{N} \sum_{j=1}^{N} e_{i}^{T} a_{i j} g\left(e_{j}-e_{i}, t\right) .
\end{aligned}
$$

According to Lemma 4 and Assumption 5, one has

$$
\begin{aligned}
\dot{V} \leq & \eta \sum_{i=1}^{N} e_{i}^{T} e_{i}-\alpha \sum_{i=1}^{N} \sum_{j=1}^{N} e_{i}^{T} l_{i j} e_{j}-\alpha h_{i} \sum_{i=1}^{N} e_{i}^{T} e_{i} \\
& -2 \sum_{i=1}^{N} \sum_{j=1}^{N} a_{i j}\left(e_{j}-e_{i}\right)^{T} g\left(e_{j}-e_{i}, t\right) \\
\leq & \eta \sum_{i=1}^{N} e_{i}^{T} e_{i}-\alpha \sum_{i=1}^{N} \sum_{j=1}^{N} e_{i}^{T} l_{i j} e_{j}-\alpha h_{i} \sum_{i=1}^{N} e_{i}^{T} e_{i} \\
& +2 \sum_{i=1}^{N} \sum_{j=1}^{N} a_{i j}\left\|e_{j}-e_{i}\right\|_{2}\left\|g\left(e_{j}-e_{i}, t\right)\right\|_{2} \\
\leq & \eta \sum_{i=1}^{N} e_{i}^{T} e_{i}-\alpha \sum_{i=1}^{N} \sum_{j=1}^{N} e_{i}^{T} l_{i j} e_{j}-\alpha h_{i} \sum_{i=1}^{N} e_{i}^{T} e_{i} \\
& +2 \sum_{i=1}^{N} \sum_{j=1}^{N} a_{i j}\left\|e_{j}-e_{i}\right\|_{2} g_{0}\left(\left\|e_{j}-e_{i}\right\|_{2}\right) \\
\leq & \eta \sum_{i=1}^{N} e_{i}^{T} e_{i}-\alpha \sum_{i=1}^{N} \sum_{j=1}^{N} e_{i}^{T} l_{i j} e_{j}-\alpha h_{i} \sum_{i=1}^{N} e_{i}^{T} e_{i} \\
& +2 M_{\delta} \sum_{i=1}^{N} \sum_{j=1}^{N} a_{i j}\left\|e_{j}-e_{i}\right\|_{2}^{2} .
\end{aligned}
$$

According to Lemma 7, we get

$$
\begin{aligned}
\dot{V} \leq & \eta \sum_{i=1}^{N} e_{i}^{T} e_{i}-\alpha \sum_{i=1}^{N} \sum_{j=1}^{N} e_{i}^{T} l_{i j} e_{j}-\alpha h_{i} \sum_{i=1}^{N} e_{i}^{T} e_{i} \\
& +4 M_{\delta} \sum_{i=1}^{N} \sum_{j=1}^{N} l_{i j} e_{i}^{T} e_{j} \\
\leq & \eta \sum_{i=1}^{N} e_{i}^{T} e_{i}-\alpha \sum_{i=1}^{N} \sum_{j=1}^{N} e_{i}^{T} l_{i j} e_{j}-\alpha h_{i} \sum_{i=1}^{N} e_{i}^{T} e_{i}
\end{aligned}
$$




$$
\begin{aligned}
& +4 M_{\delta} \sum_{i=1}^{N} \sum_{j=1}^{N} l_{i j} e_{i}^{T} e_{j}+4 M_{\delta} \alpha h_{i} \sum_{i=1}^{N} e_{i}^{T} e_{i} \\
= & -\left(\alpha-4 M_{\delta}\right) e^{T}(L+H) e+\eta e^{T} e .
\end{aligned}
$$

According to (12), one has $\dot{V}<0$, which means that the error systems (9) are locally asymptotically stable. Then $x_{i}(t) \longrightarrow$ $x_{0}(t)$, for $t \longrightarrow \infty$. This means that Theorem 9 holds.

3.2. Robust Leader-Following Practical Consensus. In this subsection, robust consensus for nonlinear multiagent systems is investigated in the case where $D_{0} \neq 0$ in Assumption 5 .

Theorem 10. Consider a networked multiagent system with $N$ followers and a virtual leader, in which the dynamics are described as (1)-(4). Suppose that Assumptions 1-5 hold and the constant $D_{0} \neq 0$ in Assumption 5. Under the consensus protocol (5), robust practical consensus of system (1) can be achieved if the following two conditions hold:

(i) There exists at least one pinned node.

(ii) For a given positive constant $\delta$,

$$
\beta_{\delta}=\left(\alpha-\left(2+4 M_{\delta}\right)\right) \lambda_{1}>\eta,
$$

where $\lambda_{1}$ is the minimal eigenvalue of $(L+H)$.

Proof. Propose the Lyapunov candidate function as

$$
V=\frac{1}{2} \sum_{i=1}^{N} e_{i}^{T} e_{i}
$$

According to (15), one can obtain

$$
\begin{aligned}
\dot{V} \leq & \eta \sum_{i=1}^{N} e_{i}^{T} e_{i}-\alpha \sum_{i=1}^{N} \sum_{j=1}^{N} e_{i}^{T} l_{i j} e_{j}-\alpha h_{i} \sum_{i=1}^{N} e_{i}^{T} e_{i} \\
& -2 \sum_{i=1}^{N} \sum_{j=1}^{N} a_{i j}\left(e_{j}-e_{i}\right)^{T} g\left(e_{j}-e_{i}, t\right) \\
\leq & \eta \sum_{i=1}^{N} e_{i}^{T} e_{i}-\alpha \sum_{i=1}^{N} \sum_{j=1}^{N} e_{i}^{T} l_{i j} e_{j}-\alpha h_{i} \sum_{i=1}^{N} e_{i}^{T} e_{i} \\
& +\sum_{i=1}^{N} \sum_{j=1}^{N} a_{i j}\left(e_{j}-e_{i}\right)^{T}\left(e_{j}-e_{i}\right) \\
& +\sum_{i=1}^{N} \sum_{j=1}^{N} a_{i j}\left(g\left(e_{j}-e_{i}, t\right)\right)^{T} g\left(e_{j}-e_{i}, t\right) .
\end{aligned}
$$

According to Lemma 7 and Assumption 5, we get

$$
\begin{aligned}
\dot{V} \leq & \eta \sum_{i=1}^{N} e_{i}^{T} e_{i}-\alpha \sum_{i=1}^{N} \sum_{j=1}^{N} e_{i}^{T} l_{i j} e_{j}-\alpha h_{i} \sum_{i=1}^{N} e_{i}^{T} e_{i} \\
& +2 \sum_{i=1}^{N} \sum_{j=1}^{N} l_{i j} e_{i}^{T} e_{j}
\end{aligned}
$$

$$
\begin{aligned}
& \quad+\sum_{i=1}^{N} \sum_{j=1}^{N} a_{i j}\left(g_{0}\left(\left\|e_{j}-e_{i}\right\|_{2}\right)+D_{0}\right)^{2} \\
& \leq \eta \sum_{i=1}^{N} e_{i}^{T} e_{i}-\alpha \sum_{i=1}^{N} \sum_{j=1}^{N} e_{i}^{T} l_{i j} e_{j}-\alpha h_{i} \sum_{i=1}^{N} e_{i}^{T} e_{i} \\
& +2 \sum_{i=1}^{N} \sum_{j=1}^{N} l_{i j} e_{i}^{T} e_{j}+2 \sum_{i=1}^{N} \sum_{j=1}^{N} a_{i j} g_{0}\left(\left\|e_{j}-e_{i}\right\|_{2}\right)^{2} \\
& +2 \sum_{i=1}^{N} \sum_{j=1}^{N} D_{0}^{2} .
\end{aligned}
$$

According to Remark 6 and Assumption 5, one has

$$
\begin{aligned}
\dot{V} \leq & \eta \sum_{i=1}^{N} e_{i}^{T} e_{i}-\alpha \sum_{i=1}^{N} \sum_{j=1}^{N} e_{i}^{T} l_{i j} e_{j}-\alpha h_{i} \sum_{i=1}^{N} e_{i}^{T} e_{i} \\
& +2 \sum_{i=1}^{N} \sum_{j=1}^{N} l_{i j} e_{i}^{T} e_{j}+2 M_{\delta} \sum_{i=1}^{N} \sum_{j=1}^{N} a_{i j}\left\|e_{j}-e_{i}\right\|_{2}^{2} \\
& +2 N(N-1) D_{0}^{2} \\
= & \eta \sum_{i=1}^{N} e_{i}^{T} e_{i}-\alpha \sum_{i=1}^{N} \sum_{j=1}^{N} e_{i}^{T} l_{i j} e_{j}-\alpha h_{i} \sum_{i=1}^{N} e_{i}^{T} e_{i} \\
& +\left(2+4 M_{\delta}\right) \sum_{i=1}^{N} \sum_{j=1}^{N} l_{i j} e_{i}^{T} e_{j}+2 N(N-1) D_{0}^{2} \\
\leq & e^{T}\left(\left(\eta I-\left(\alpha-\left(2+4 M_{\delta}\right)\right)(L+H)\right) \otimes I_{n}\right) e \\
& +2 N(N-1) D_{0}^{2} \\
\leq & -\left(\beta_{\delta}-\eta\right) V+2 N(N-1) D_{0}^{2} \\
= & -\left(\beta_{\delta}-\eta\right)\left(V-\frac{2 N(N-1) D_{0}^{2}}{\beta_{\delta}-\eta}\right)
\end{aligned}
$$

Since $\beta_{\delta}>\eta, \dot{V}<0$ if $V>2 N(N-1) D_{0}^{2} /\left(\beta_{\delta}-\eta\right)$, which means that all the state errors will enter into the set $S=\{V \leq$ $\left.2 N(N-1) D_{0}^{2} /\left(\beta_{\delta}-\eta\right)\right\}$. This means that Theorem 10 holds.

\section{Robust Leaderless Consensus for Nonlinear Multiagent Systems with Uncertainty and Disturbance}

In this section, the robust leaderless consensus of nonlinear multiagent systems with bounded channel disturbance is investigated. Consider the following consensus protocol:

$$
\begin{aligned}
& u_{i}=\sum_{j \in \mathscr{N}_{i}} a_{i j}(t)\left[\alpha\left(x_{j}-x_{i}\right)+g\left(t, x_{j}-x_{i}\right)\right] \\
& \\
& i=1,2, \ldots, N,
\end{aligned}
$$


where $\alpha>0$ and $g: R \times R^{n} \longrightarrow R^{n}$ are defined the same way as Section 3. Denoting $\bar{x}=\sum_{i=1}^{N} x_{i} / N, \bar{x}=\left(\sum_{i=1}^{N} f\left(t, x_{i}\right)+\right.$ $\left.\sum_{i=1}^{N} u_{i}\right) / N$. Since the Laplacian matrix $L$ is symmetric and zero-row-sum, one has $\sum_{j=1}^{N} a_{i j}(t)\left(x_{j}-x_{i}\right)=\sum_{j=1}^{N} l_{i j}(t) x_{j}=$ 0 ; then $\sum_{i=1}^{N} \sum_{j=1}^{N} a_{i j}(t)\left(x_{j}-x_{i}\right)=-\sum_{i=1}^{N} \sum_{j=1}^{N} l_{i j}(t) x_{j}=0$. Furthermore, noting that $g\left(t, x_{j}-x_{i}\right)=-g\left(t, x_{i}-x_{j}\right)$, one can conclude that $\sum_{i=1}^{N} \sum_{j=1}^{N} a_{i j}(t) g\left(t, x_{j}-x_{i}\right)=0$. Then $\sum_{i=1}^{N} u_{i}=$ $\sum_{i=1}^{N} \sum_{j=1}^{N} a_{i j}(t)\left(\alpha\left(x_{j}-x_{i}\right)+g\left(t, x_{j}-x_{i}\right)\right)=0$. It follows that $\dot{\bar{x}}=0$. Let $\tilde{x}_{i}=x_{i}-\bar{x}$; according to (9), the error system can be described as

$$
\begin{aligned}
\dot{e}_{i}(t)= & f\left(t, x_{i}\right)-\frac{\sum_{j=1}^{N} f\left(t, x_{j}\right)}{N}-\sum_{j \in \mathcal{N}_{i}} \alpha l_{i j} e_{j} \\
& +\sum_{j \in \mathcal{N}_{i}} a_{i j} g\left(e_{j}-e_{i}, t\right), \quad i=1,2, \ldots, N .
\end{aligned}
$$

4.1. Robust Leaderless Consensus. In this subsection, robust consensus for nonlinear multiagent systems is investigated in the case where $D_{0}=0$ in Assumption 5 .

Theorem 11. Consider a networked multiagent system with $N$ follower agents, in which the dynamics are described as (1). Suppose that Assumptions 1-5 hold and the constant $D_{0}=0$ in Assumption 5. Under the consensus protocol (22), robust consensus of system (1) can be achieved if

$$
\beta_{\delta}=\left(\alpha-M_{\delta}\right) \lambda_{1}>5 \eta
$$

where $\lambda_{2}(L)$ is the minimal eigenvalue of $L$ and $\delta$ is a given positive constant.

Proof. Choose the Lyapunov candidate function as

$$
V=\frac{1}{2} \sum_{i=1}^{N} e_{i}^{T} e_{i}
$$

Differentiating $V$ with respect to $t$ along (23), one can obtain

$$
\begin{aligned}
\dot{V}= & \sum_{i=1}^{N} e_{i}^{T} \dot{e}_{i} \\
= & \sum_{i=1}^{N} e_{i}^{T}\left(f\left(t, x_{i}\right)-\frac{\sum_{j=1}^{N} f\left(t, x_{j}\right)}{N}\right) \\
& -\sum_{i=1}^{N} e_{i}^{T}\left(\sum_{j=1}^{N} \alpha l_{i j} e_{j}-\sum_{j=1}^{N} a_{i j} g\left(x_{j}-x_{i}, t\right)\right) \\
\leq & \eta \sum_{i=1}^{N}\left\|e_{i}\right\|_{2}\left\|e_{i}-e_{j}\right\|_{2}-\alpha \sum_{i=1}^{N} \sum_{j=1}^{N} e_{i}^{T} l_{i j} e_{j} \\
& +\sum_{i=1}^{N} \sum_{j=1}^{N} e_{i}^{T} a_{i j} g\left(e_{j}-e_{i}, t\right)
\end{aligned}
$$

$$
\begin{array}{r}
\leq 5 \eta \sum_{i=1}^{N} e_{i}^{T} e_{i}-\alpha \sum_{i=1}^{N} \sum_{j=1}^{N} e_{i}^{T} l_{i j} e_{j} \\
+\sum_{i=1}^{N} \sum_{j=1}^{N} e_{i}^{T} a_{i j} g\left(e_{j}-e_{i}, t\right) .
\end{array}
$$

According to (15)-(16), one has

$$
\begin{aligned}
\dot{V} \leq & 5 \eta \sum_{i=1}^{N} e_{i}^{T} e_{i}-\alpha \sum_{i=1}^{N} \sum_{j=1}^{N} e_{i}^{T} l_{i j} e_{j} \\
& +2 M_{\delta} \sum_{i=1}^{N} \sum_{j=1}^{N} a_{i j}\left\|e_{j}-e_{i}\right\|_{2}^{2} \\
\leq & \eta \sum_{i=1}^{N} e_{i}^{T} e_{i}-\alpha \sum_{i=1}^{N} \sum_{j=1}^{N} e_{i}^{T} l_{i j} e_{j}+4 M_{\delta} \sum_{i=1}^{N} \sum_{j=1}^{N} l_{i j} e_{i}^{T} e_{j} \\
= & -\left(\alpha-4 M_{\delta}\right) e^{T} L e+5 \eta e^{T} e .
\end{aligned}
$$

Noting that $\mathbf{1}^{T} \widetilde{e}=\sum_{i=1}^{N}\left(x_{i}-\sum_{i=1}^{N} x_{i} / N\right)=0$, according to Lemma $4, e^{T} L e \geq e^{T} \lambda_{2}(L) e$. Then, following with (24), one has $\dot{V}<0$, which means that the error systems (23) are locally asymptotically stable. Then $x_{i}(t) \longrightarrow \bar{x}(t)$, for $t \longrightarrow \infty$. This means that Theorem 11 holds.

4.2. Robust Leaderless Practical Consensus. In this subsection, robust consensus for nonlinear multiagent systems is investigated in the case where $D_{0} \neq 0$ in Assumption 5 .

Theorem 12. Consider a networked multiagent system with $N$ follower agents, in which the dynamics are described as (1). Suppose that Assumptions 1-5 hold and the constant $D_{0} \neq 0$ in Assumption 5. Under the consensus protocol (22), robust practical consensus of system (1) can be achieved if

$$
\beta_{\delta}=\left(\alpha-\left(2+4 M_{\delta}\right)\right) \lambda_{2}>5 \eta,
$$

where $\lambda_{2}(L)$ is the minimal eigenvalue of $L$ and $\delta$ is a given positive constant.

Proof. Propose the Lyapunov candidate function as

$$
V=\frac{1}{2} \sum_{i=1}^{N} e_{i}^{T} e_{i} .
$$

According to (26), one can obtain

$$
\begin{aligned}
\dot{V} \leq & \sum_{i=1}^{N} e_{i}^{T}\left(f\left(t, x_{i}\right)-\frac{\sum_{j=1}^{N} f\left(t, x_{j}\right)}{N}\right) \\
& -\alpha \sum_{i=1}^{N} \sum_{j=1}^{N} e_{i}^{T} l_{i j} e_{j} \\
& -2 \sum_{i=1}^{N} \sum_{j=1}^{N} a_{i j}\left(e_{j}-e_{i}\right)^{T} g\left(e_{j}-e_{i}, t\right)
\end{aligned}
$$




$$
\begin{aligned}
\leq & 5 \eta \sum_{i=1}^{N} e_{i}^{T} e_{i}-\alpha \sum_{i=1}^{N} \sum_{j=1}^{N} e_{i}^{T} l_{i j} e_{j} \\
& +\sum_{i=1}^{N} \sum_{j=1}^{N} a_{i j}\left(e_{j}-e_{i}\right)^{T}\left(e_{j}-e_{i}\right) \\
& +\sum_{i=1}^{N} \sum_{j=1}^{N} a_{i j}\left(g\left(e_{j}-e_{i}, t\right)\right)^{T} g\left(e_{j}-e_{i}, t\right) \\
\leq & 5 \eta \sum_{i=1}^{N} e_{i}^{T} e_{i}-(\alpha-2) \sum_{i=1}^{N} \sum_{j=1}^{N} e_{i}^{T} l_{i j} e_{j} \\
& +\sum_{i=1}^{N} \sum_{j=1}^{N} a_{i j}\left(g_{0}\left(\left\|e_{j}-e_{i}\right\|_{2}\right)+D_{0}\right)^{2} \\
& +2 \sum_{i=1}^{N} \sum_{j=1}^{N} a_{i j} g_{0}\left(\left\|e_{j}-e_{i}\right\|_{2}\right)^{2}+2 \sum_{i=1}^{N} \sum_{j=1}^{N} D_{0}^{2} .
\end{aligned}
$$

According to Remark 6 and Assumption 5, one has

$$
\begin{aligned}
\dot{V} \leq & 5 \eta \sum_{i=1}^{N} e_{i}^{T} e_{i}-(\alpha-2) \sum_{i=1}^{N} \sum_{j=1}^{N} e_{i}^{T} l_{i j} e_{j} \\
& +2 M_{\delta} \sum_{i=1}^{N} \sum_{j=1}^{N} a_{i j}\left\|e_{j}-e_{i}\right\|_{2}^{2}+2 N(N-1) D_{0}^{2} \\
= & 5 \eta \sum_{i=1}^{N} e_{i}^{T} e_{i}-(\alpha-2) \sum_{i=1}^{N} \sum_{j=1}^{N} e_{i}^{T} l_{i j} e_{j} \\
& +4 M_{\delta} \sum_{i=1}^{N} \sum_{j=1}^{N} l_{i j} e_{i}^{T} e_{j}+2 N(N-1) D_{0}^{2} \\
\leq & e^{T}\left(\left(5 \eta I-\left(\alpha-\left(2+4 M_{\delta}\right)\right) L\right) \otimes I_{n}\right) e \\
& +2 N(N-1) D_{0}^{2} \\
\leq & -\left(\beta_{\delta}-\eta\right) V+2 N(N-1) D_{0}^{2} \\
= & \left(\beta_{\delta}-5 \eta\right)\left(V-\frac{2 N(N-1) D_{0}^{2}}{\beta_{\delta}-5 \eta}\right)
\end{aligned}
$$

Since $\beta_{\delta}>5 \eta, \dot{V}<0$ if $V>2 N(N-1) D_{0}^{2} /\left(\beta_{\delta}-5 \eta\right)$, which means that all the state errors will enter into the set $S=\{V \leq$ $\left.2 N(N-1) D_{0}^{2} /\left(\beta_{\delta}-\eta\right)\right\}$. This means that Theorem 12 holds.

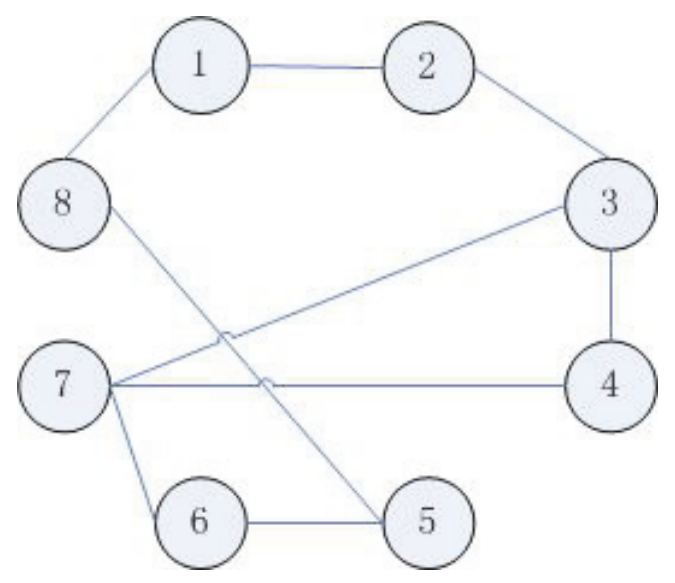

FIGURE 1: Topology graph G.

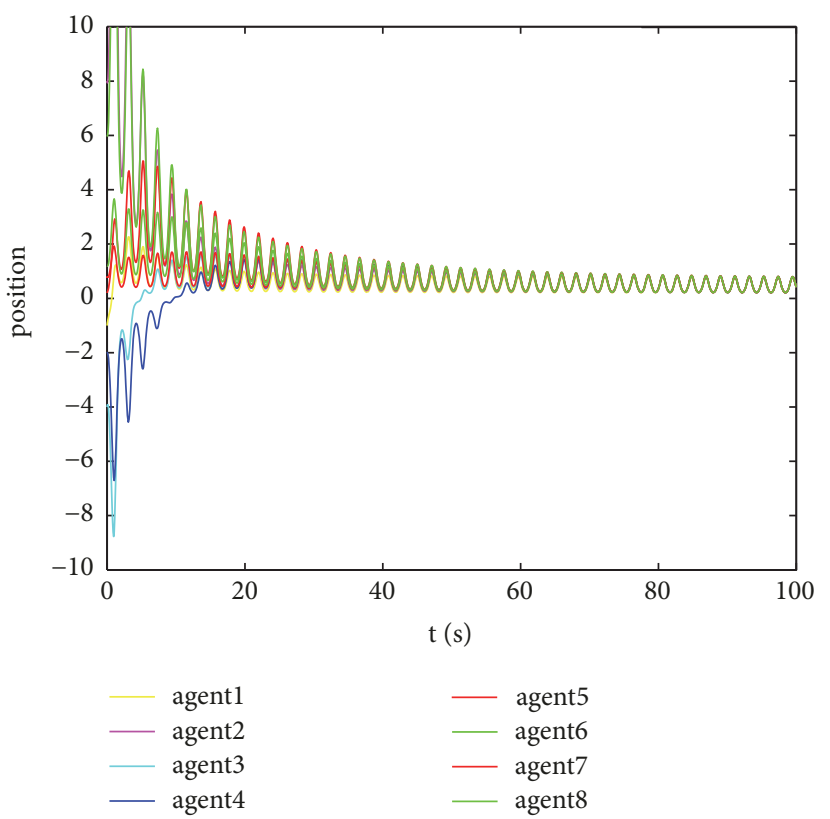

FigURE 2: Leader-following consensus of state trajectories.

\section{Simulations}

Consider a network with 8 nodes and a virtual leader. The topology is described as Figure 1. We consider both complete consensus and practical consensus for nonlinear first-order multiagent systems for the cases where $D_{0}=0$ and $D_{0} \neq 0$, respectively. The dynamics of each agent are described as $\dot{x}_{i}=$ $2 x_{i} \sin 3 t+u_{i}$.

For the complete consensus case, choosing the nonlinear disturbance function is chosen as $g(x, t)=0.02 \sin x$; according to Figures 2 and 4, we can know that the consensus for the proposed network can be achieved. For the practical consensus case, choosing the nonlinear disturbance function is chosen as $g(x, t)=0.02 \sin x+0.2 \cos t$; according to Figures 3 and 5, it can be found that there is a tiny error in the trajectories, which means that the practical consensus is achieved. 


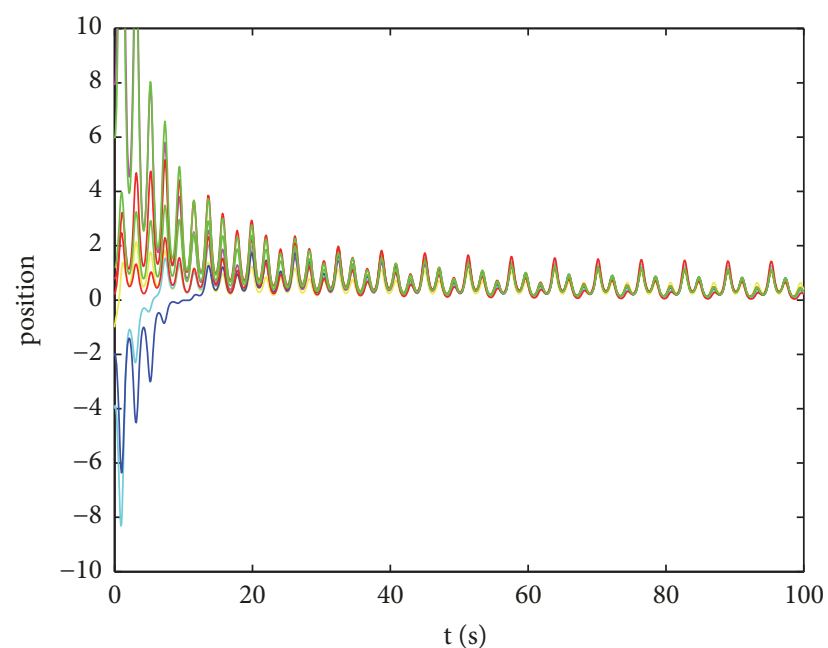

$\begin{array}{rr}\text { agent1 } & - \text { agent5 } \\ \text { agent } 2 & - \text { agent6 } \\ \text { agent3 } & - \text { agent7 } \\ \text { agent } 4 & - \text { agent8 }\end{array}$

FIGURE 3: Leader-following practical consensus of state trajectories.

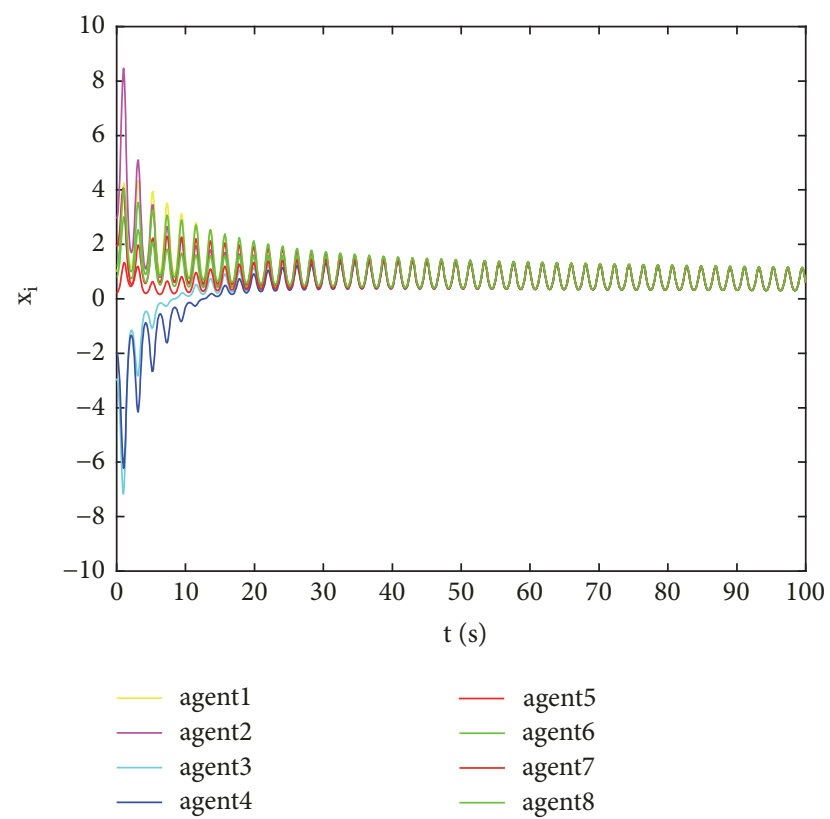

FIGURE 4: Leaderless consensus of state trajectories.

\section{Conclusions}

In this paper, both the robust consensus and the practical consensus problem are investigated for nonlinear multiagent systems with uncertainty and disturbance. The uncertainty and disturbance function is piecewise continuous and is described as $g(x, t)$, satisfying $\|g(x, t)\|_{2} \leq g_{0}\left(\|x\|_{2}\right)+D_{0}$. Both complete consensus and practical consensus condition are obtained and the analysis is presented by using hybrid tools from matrix theory and Lyapunov stability theory.

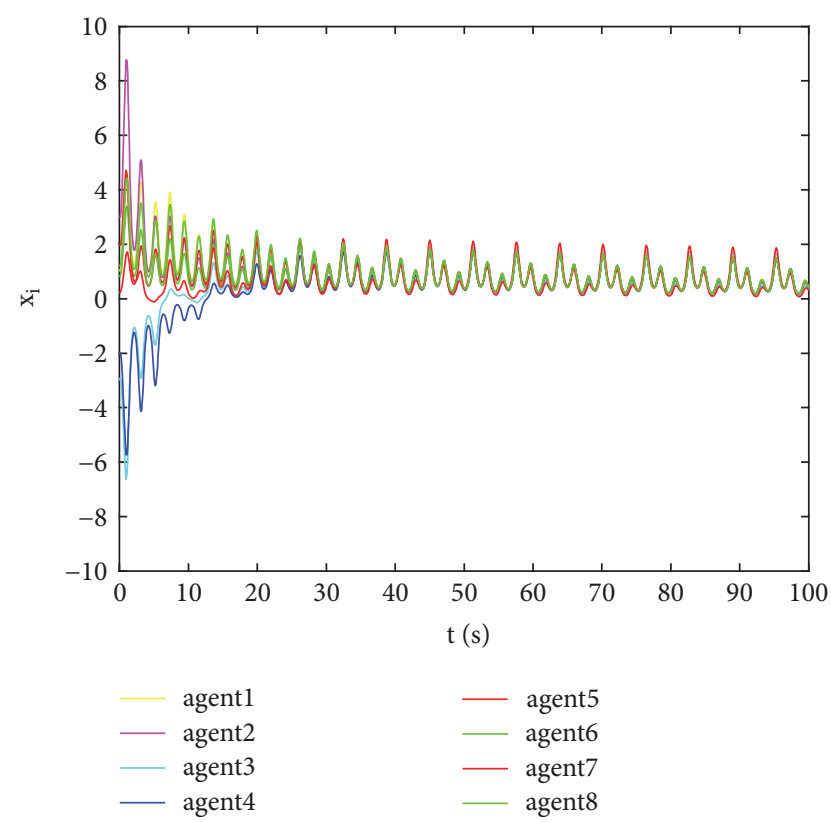

FIGURE 5: Leader-following practical consensus of state trajectories.

\section{Data Availability}

The data used to support the findings of this study are available from the corresponding author upon request.

\section{Conflicts of Interest}

The authors declare that there are no conflicts of interest regarding the publication of this paper.

\section{Acknowledgments}

This work is supported by the National Natural Science Foundation of China (Grant no. 61703154), Natural Science Foundation of Guangxi (2014 GXNSFBA118268), Outstanding Young Project of the Department of Education in Hunan Province (16B070), Science and Technology Research Foundation of Guangxi Universities (201204LX339), China Postdoctoral Science Foundation (2016M602298), and MOE Key Laboratory of Image Processing and Intelligence Control (IPIC2017-04).

\section{References}

[1] R. Olfati-Saber, J. A. Fax, and R. M. Murray, "Consensus and cooperation in networked multi-agent systems," Proceedings of the IEEE, vol. 95, no. 1, pp. 215-233, 2007.

[2] W. Ren and R. W. Beard, "Consensus seeking in multiagent systems under dynamically changing interaction topologies," IEEE Transactions on Automatic Control, vol. 50, no. 5, pp. 655-661, 2005.

[3] H. Su, H. Wu, and X. Chen, "Observer-based discrete-time nonnegative edge synchronization of networked systems," IEEE Transactions on Neural Networks and Learning Systems, vol. 28, no. 10, pp. 2446-2455, 2017. 
[4] H. Su, H. Wu, X. Chen, and M. Z. Chen, "Positive Edge Consensus of Complex Networks," IEEE Transactions on Systems, Man, and Cybernetics: Systems, pp. 1-9.

[5] Z. Ji, H. Lin, and H. Yu, "Protocols design and uncontrollable topologies construction for multi-agent networks," IEEE Transactions on Automatic Control, vol. 60, no. 3, pp. 781-786, 2015.

[6] Y. Zhang, Z. Wang, and L. Ma, "Variance-constrained state estimation for networked multi-rate systems with measurement quantization and probabilistic sensor failures," International Journal of Robust and Nonlinear Control, vol. 26, no. 16, pp. 3507-3523, 2016.

[7] C. Xu, Y. Zheng, H. Su, and H. . Wang, "Containment control for coupled harmonic oscillators with multiple leaders under directed topology," International Journal of Control, vol. 88, no. 2, pp. 248-255, 2015.

[8] H. Q. Li, X. F. Liao, and T. W. Huang, "Second-order locally dynamical consensus of multiagent systems with arbitrarily fast switching directed topologies," IEEE Transactions on Systems, Man, and Cybernetics: Systems, vol. 43, no. 6, pp. 1343-1353, 2013.

[9] H. Su, M. Z. Q. Chen, J. Lam, and Z. Lin, "Semi-global leaderfollowing consensus of linear multi-agent systems with input saturation via low gain feedback," IEEE Transactions on Circuits and Systems I: Regular Papers, vol. 60, no. 7, pp. 1881-1889, 2013.

[10] L. Cheng, Y. Wang, Z.-G. Hou, M. Tan, and Z. Cao, "Sampled-data based average consensus of second-order integral multi-agent systems: switching topologies and communication noises," Automatica, vol. 49, no. 5, pp. 1458-1464, 2013.

[11] Z. Li, W. Ren, X. Liu, and M. Fu, "Consensus of multi-agent systems with general linear and lipschitz nonlinear dynamics using distributed adaptive protocols," IEEE Transactions on Automatic Control, vol. 58, no. 7, pp. 1786-1791, 2013.

[12] Z. Li, Z. Duan, and F. L. Lewis, "Distributed robust consensus control of multi-agent systems with heterogeneous matching uncertainties," Automatica, vol. 50, no. 3, pp. 883-889, 2014.

[13] W. W. Yu, G. R. Chen, M. Cao, and J. Kurths, "Second-order consensus for multiagent systems with directed topologies and nonlinear dynamics," IEEE Transactions on Systems, Man, and Cybernetics, Part B: Cybernetics, vol. 40, no. 3, pp. 881-891, 2010.

[14] H. Du, S. Li, and P. Shi, "Robust consensus algorithm for second-order multi-agent systems with external disturbances," International Journal of Control, vol. 85, no. 12, pp. 1913-1928, 2012.

[15] G. Hu, "Robust consensus tracking of a class of second-order multi-agent dynamic systems," Systems \& Control Letters, vol. 61, no. 1, pp. 134-142, 2012.

[16] T. Li and J.-F. Zhang, "Mean square average-consensus under measurement noises and fixed topologies: necessary and sufficient conditions," Automatica, vol. 45, no. 8, pp. 1929-1936, 2009.

[17] F. Chen, L. Xiang, W. Lan, and G. Chen, "Coordinated tracking in mean square for a multi-agent system with noisy channels and switching directed network topologies," IEEE Transactions on Circuits and Systems II: Express Briefs, vol. 59, no. 11, pp. 835839, 2012.

[18] L. Cheng, Z.-G. Hou, M. Tan, and X. Wang, "Necessary and sufficient conditions for consensus of double-integrator multiagent systems with measurement noises," IEEE Transactions on Automatic Control, vol. 56, no. 8, pp. 1958-1963, 2011.

[19] H. Yang, L. Guo, and C. Han, "Robust consensus of multi-agent systems with uncertain exogenous disturbances," Communications in Theoretical Physics, vol. 56, no. 6, pp. 1161-1166, 2011.
[20] C. Xu, Y. Zheng, H. Su, and H.-B. Zeng, "Containment for linear multi-agent systems with exogenous disturbances," Neurocomputing, vol. 160, pp. 206-212, 2015.

[21] Z. Y. Meng, Z. L. Lin, and W. Ren, "Robust cooperative tracking for multiple non-identical second-order nonlinear systems," Automatica, vol. 49, no. 8, pp. 2363-2372, 2013.

[22] H. S. Su, M. Z. Q. Chen, and G. R. Chen, "Robust semi-global coordinated tracking of linear multi-agent systems with input saturation," International Journal of Robust and Nonlinear Control, vol. 25, no. 14, pp. 2375-2390, 2015.

[23] C. Xu, Y. Zhao, B. Qin, and H. Zhang, "Adaptive synchronization of coupled harmonic oscillators under switching topology," Journal of the Franklin Institute, In press.

[24] H. Su, Z. Rong, M. Z. Q. Chen, X. Wang, G. Chen, and H. Wang, "Decentralized adaptive pinning control for cluster synchronization of complex dynamical networks," IEEE Transactions on Systems, Man, and Cybernetics, Part B: Cybernetics, vol. 43, no. 1, pp. 394-399, 2013. 


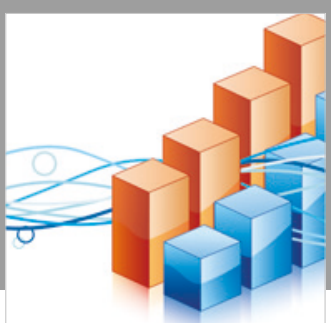

Advances in

Operations Research

\section{-n-m}
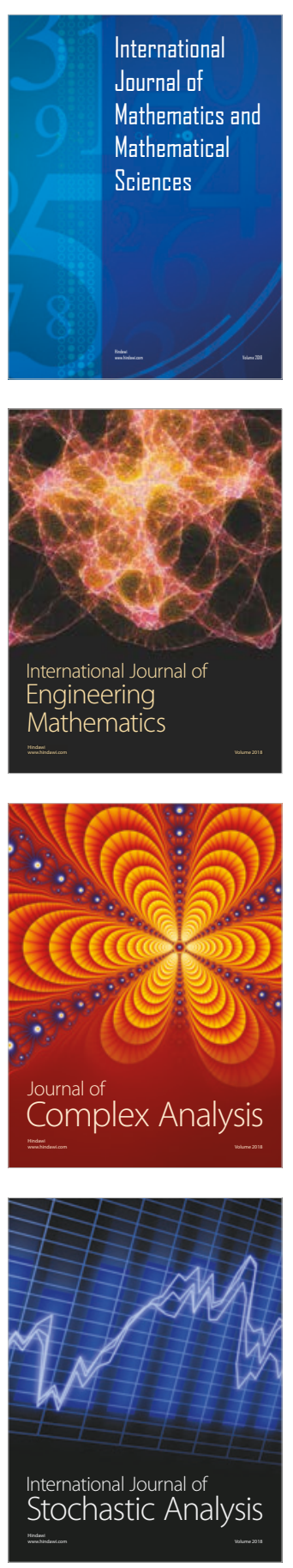
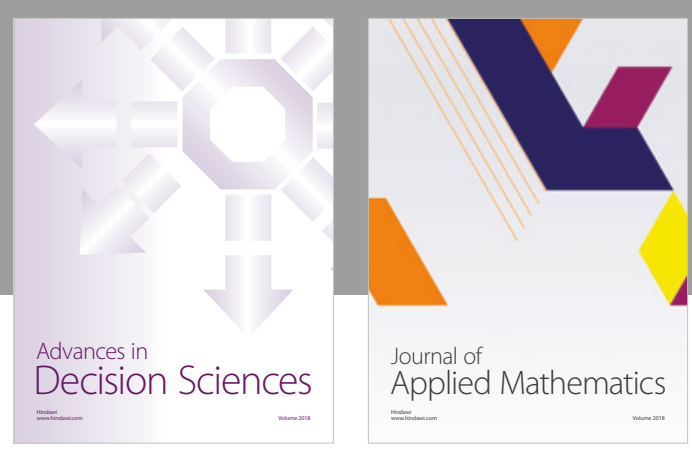

Journal of

Applied Mathematics
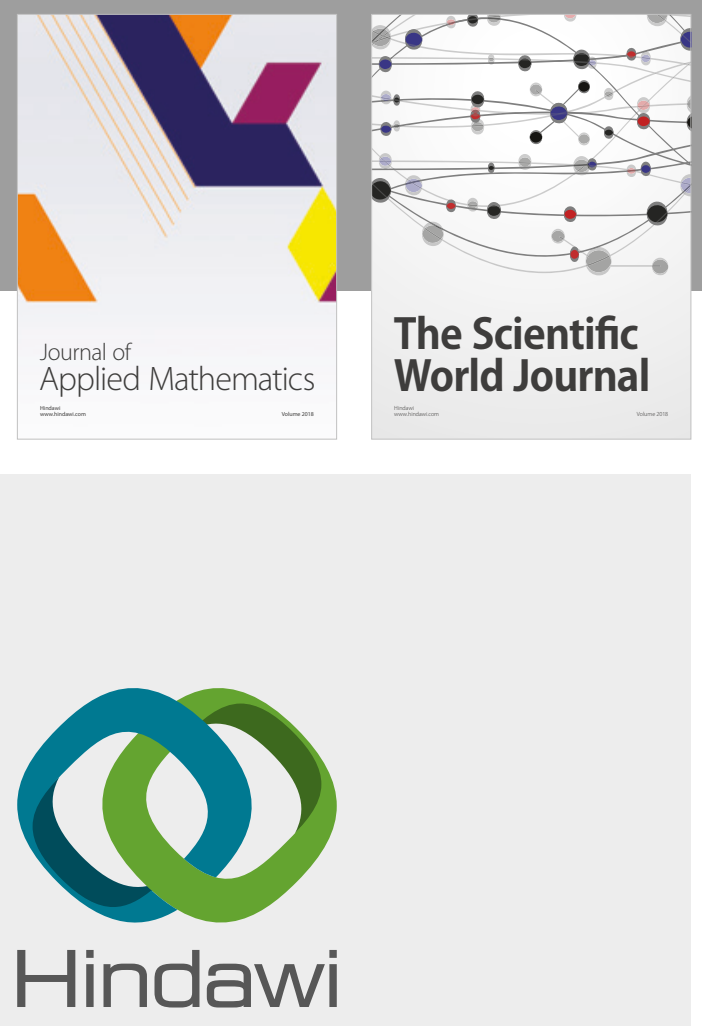

Submit your manuscripts at

www.hindawi.com

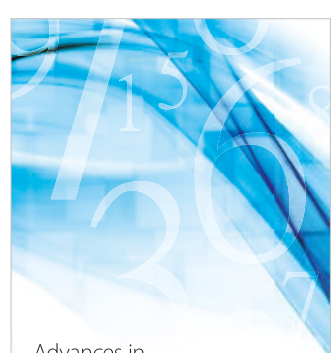

Advances in
Numerical Analysis
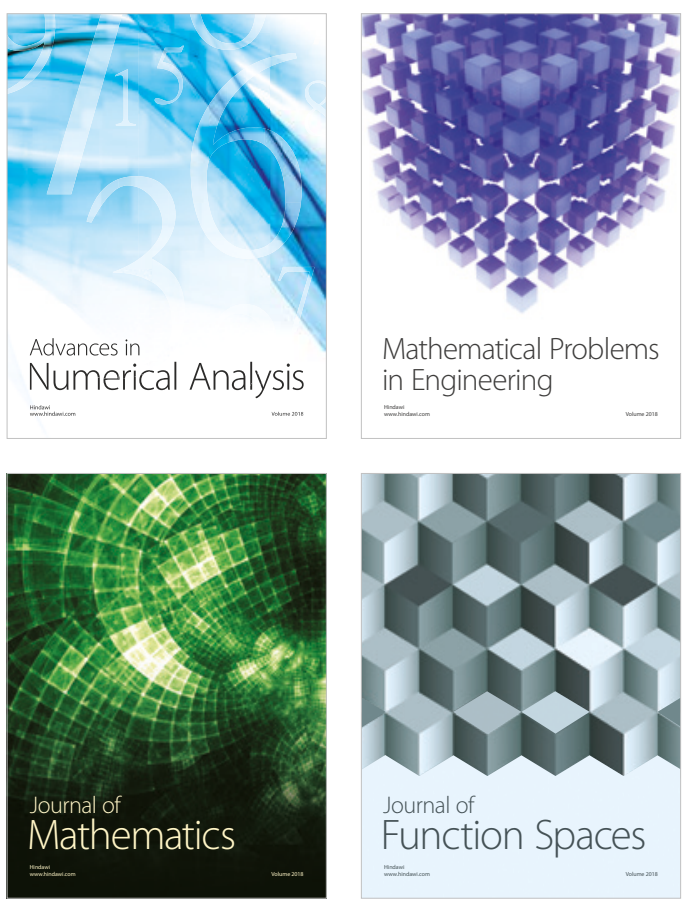

Mathematical Problems in Engineering

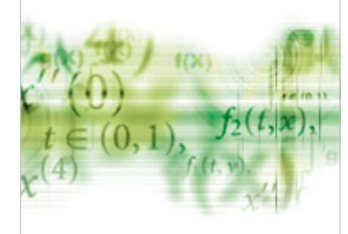

International Journal of

Differential Equations

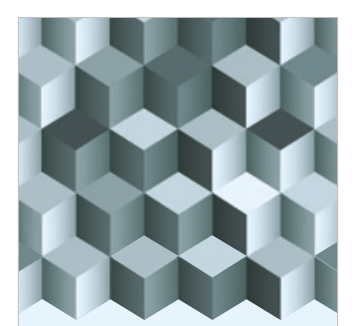

Journal of

Function Spaces

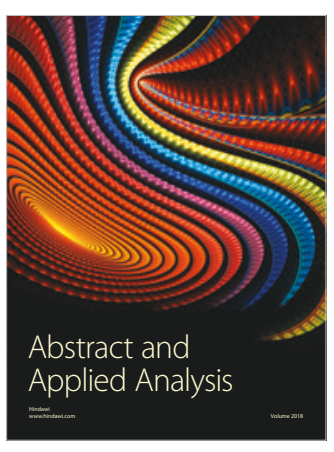

The Scientific

World Journal

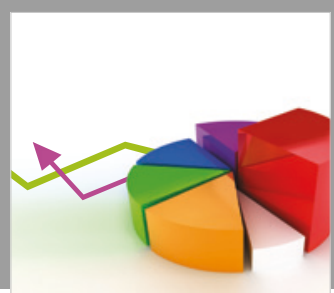

Journal of

Probability and Statistics
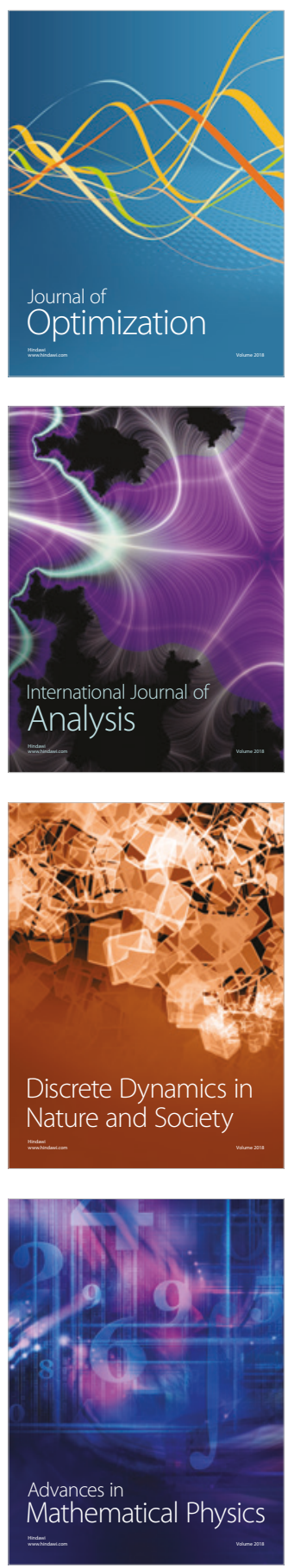